\title{
Strain-driven criticality underlies nonlinear mechanics of fibrous networks
}

\author{
A. Sharma, ${ }^{1,2}$ A. J. Licup, ${ }^{1}$ R. Rens, ${ }^{1}$ M. Vahabi, ${ }^{1}$ K. A. Jansen,${ }^{3,4}$ G. H. Koenderink, ${ }^{3}$ and F. C. MacKintosh ${ }^{1,5}$ \\ ${ }^{1}$ Department of Physics and Astronomy, VU University, 1081 NL Amsterdam, The Netherlands \\ ${ }^{2}$ Department of Physics, University of Fribourg, CH-1700 Fribourg, Switzerland \\ ${ }^{3}$ FOM Institute AMOLF, Science Park 104, 1098 XG Amsterdam, The Netherlands \\ ${ }^{4}$ Wellcome Trust Centre for Cell-Matrix Research, Faculty of Biology, Medicine \\ and Health, University of Manchester, Manchester M13 9PT, United Kingdom \\ ${ }^{5}$ Departments of Chemical \& Biomolecular Engineering, Chemistry, Physics \& Astronomy, Rice University, Houston, Texas 77005, USA
}

(Received 27 June 2016; published 11 October 2016)

\begin{abstract}
Networks with only central force interactions are floppy when their average connectivity is below an isostatic threshold. Although such networks are mechanically unstable, they can become rigid when strained. It was recently shown that the transition from floppy to rigid states as a function of simple shear strain is continuous, with hallmark signatures of criticality [Sharma et al., Nature Phys. 12, 584 (2016)]. The nonlinear mechanical response of collagen networks was shown to be quantitatively described within the framework of such mechanical critical phenomenon. Here, we provide a more quantitative characterization of critical behavior in subisostatic networks. Using finite-size scaling we demonstrate the divergence of strain fluctuations in the network at well-defined critical strain. We show that the characteristic strain corresponding to the onset of strain stiffening is distinct from but related to this critical strain in a way that depends on critical exponents. We confirm this prediction experimentally for collagen networks. Moreover, we find that the apparent critical exponents are largely independent of the spatial dimensionality. With subisostaticity as the only required condition, strain-driven criticality is expected to be a general feature of biologically relevant fibrous networks.
\end{abstract}

DOI: 10.1103/PhysRevE.94.042407

\section{INTRODUCTION}

Disordered filamentous networks are ubiquitous in biology. An important example of such networks is the extracellular matrix in biological tissues, which is predominantly composed of a fibrous collagen scaffold [1]. One of the most important characteristics of such networks is the coordination number or average connectivity $\langle z\rangle$. Networks with only central force interactions are unstable toward small deformation if the average connectivity is below the threshold value of $\langle z\rangle=2 d$, where $d$ is the dimensionality. This threshold is referred to as the isostatic point at which, as shown by Maxwell [2], the number of degrees of freedom are just balanced by the number of constraints, and the system is marginally stable. As the average connectivity increases beyond $2 d$, the network undergoes a phase transition marked by a continuous increase in the elasticity. Other examples of such transitions are the jamming transition [3-6] in granular materials and rigidity percolation [7-10] in disordered spring networks. Jamming exhibits signatures characteristic of both first- and second-order transitions, with discontinuous behavior of the bulk modulus and continuous variation of the shear modulus $[5,11,12]$. For networks of springs or fibers, the transition from floppy to rigid is a continuous phase transition, in both bulk and shear moduli, with critical signatures [5,7,13-16].

In a biological context, the average connectivity is almost always below the isostatic threshold. Filamentous networks typically fall in two categories, those in which network formation occurs via branching and those where crosslinking proteins connect two distinct filaments. The typical connectivity in such networks is between 3 and 4, with the former due to branching and the latter due to binary crosslinking. In fact these networks are well below both 2D and 3D isostatic thresholds $[15,17]$. Such subisostatic networks can, however, become rigid as a result of other mechanical constraints, such as fiber bending [13,15,18,19], internal stresses [20], thermal fluctuations [21], or when subjected to external strain [16,22]. Except for the external strain, other applied fields stabilize the network even in the zero strain limit, i.e., the subisostatic network becomes stable to small deformations. However, when the applied field is an external strain, the transition from floppy to rigid states occurs at a threshold strain that depends on the network structure, nature of the applied deformation, as well as the average connectivity [16]. We recently showed that sheared subisostatic networks exhibit a line of second-order transitions at a strain threshold $\gamma_{c}(z)$, for connectivities $\langle z\rangle$ well below the isostatic threshold [23].

Here we follow up on this intriguing finding of strain-driven criticality by performing a detailed study of the nonlinear mechanics under simple shear. As a hallmark signature of criticality we demonstrate the divergence of strain fluctuations in the thermodynamic limit using finite-size scaling. In Ref. [23] it was shown that the critical exponents appear to depend on the average connectivity in the network. Here we present our findings on the evolution of critical exponents in more detail.

The article is organized as follows. In Sec. II we describe the computational model used in this study. We also describe the mapping of parameters used in simulations to the experimentally relevant control variables. In Sec. III we focus on the demonstration of strain-driven criticality in disordered networks. We show the critical scaling of the order parameter close to the critical point implying the continuous transition. In this section, we also analyze the stiffness versus strain curves for finite bending rigidities in terms of a crossover function. In Sec. III B, we investigate strain fluctuations at the critical point and demonstrate their divergence in the thermodynamic limit. In Sec. IV, we derive an approximate equation describing 
the shape of the stiffness versus strain curves. We show that the derived equation can accurately describe the mechanical response measured for reconstituted collagen networks. In Sec. V, we obtain and experimentally validate scaling relation between the onset strain for stiffening and the critical strain. In Sec. VI, we show that under simple shear, the critical exponents vary with the average connectivity. We discuss our findings together with an outlook in Sec. VII.

\section{THE MODEL}

We model lattice-based networks [24-26] in 2D and 3D. Fibers are arranged on a triangular lattice (2D) or a facecentered cubic lattice (3D) of linear dimension $W$. In 2D, we randomly select two of the three fibers at each vertex on which we form a binary cross-link, i.e., enforcing local fourfold connectivity of the network in which the third fiber does not interact with the other two [24]. Similarly, in 3D, where there are six fibers crossing at a point, we randomly connect three separate pairs of fibers at each vertex with binary cross-links to enforce local fourfold connectivity [25]. In both 2D and $3 \mathrm{D}$, the average connectivity is further reduced below 4 by random dilution of bonds with a probability $(1-p)$, where $p$ is the probability that a bond exists. The resulting connectivity after dilution can be estimated as $\langle z\rangle \simeq 4 p$. All networks, by construction, are subisostatic and floppy in the absence of bending interactions [15]. The filaments are characterized by both a stretching modulus, $\mu$, and bending rigidity, $\kappa$. These define a dimensionless rigidity $\tilde{\kappa}=\kappa / \mu l^{2}$, where $l$ is the lattice spacing (mesh size) in lattice-based (Mikado) networks. In lattice-based networks we take $l=l_{0}$ where $l_{0}$ is the lattice constant. The networks are subjected to an affine simple shear strain $\gamma$ and subsequently allowed to relax by minimization of the total elastic energy. The affine shear is applied incrementally in 30 steps from $1-1000 \%$. The strain steps are exponentially spaced. In order to verify that our results are not dependent on the loading method, we have also performed step loading of the sample. We found that under step loading the elastic energy stored in the network after relaxation is the same as obtained by incremental loading. Unless otherwise stated, all the simulations are carried out on a network of size at least $W=50 l_{0}$ in $2 \mathrm{D}$ and $W=30 l_{0}$ in $3 \mathrm{D}$. The total elastic energy per unit volume, $\mathcal{H}$, is calculated using a discrete form of the extensible wormlike chain Hamiltonian [27],

$$
\mathcal{H}=\frac{1}{W^{d}} \sum_{f}\left[\frac{\mu}{2} \int_{f}\left(\frac{d l}{d s}\right)^{2} d s+\frac{\kappa}{2} \int_{f}\left|\frac{d \hat{t}}{d s}\right|^{2} d s\right],
$$

where the term in the square brackets represents the energy stored in a single fiber and the sum is performed over all the fibers in the networks. There are other choices of modeling an individual fiber such as a truss, Euler-Bernoulli, or Timoshenko beam [28,29]. The Hamiltonian in Eq. (1) captures the semiflexible nature of biopolymers with finite resistance to both tension and bending. Details about discretization of the Hamiltonian in Eq. (1) are described elsewhere [17]. The stress and modulus are obtained by taking first and second derivatives of the energy density with respect to the applied deformation, respectively. The elastic energy involves a summation over all fibers in the network and is a function of the strain $\gamma$ and the reduced bending rigidity $\tilde{\kappa}$. Since the network stiffness or tangent modulus $K=d \sigma / d \gamma$ involves the energy per unit volume, $K$ is naturally proportional to the line density $\rho$ defined as the total length of the fibers per unit volume $[19,27,30-32]$. The modulus can therefore be expressed as

$$
K=\mu \rho \mathcal{K}(\gamma, \tilde{\kappa}),
$$

where $\mathcal{K}$ is a function of the reduced bending rigidity and the applied deformation. From the computational perspective, the most relevant quantity is the function $\mathcal{K}(\gamma, \tilde{\kappa})$. Consistent with our previous studies [17,23,33], we report the modulus (stress) in units of $\mu \rho$. The line density $\rho$ is specific to the chosen network architecture, i.e., the network geometry. In lattice-based networks, $\rho_{d}=\tilde{\rho}_{d} / l_{c}^{d-1}$ with $\tilde{\rho}_{2 \mathrm{D}}=\frac{6 p}{\sqrt{3}}$ and $\tilde{\rho}_{3 \mathrm{D}}=\frac{12 p}{\sqrt{2}}$ [17]. For Mikado networks, because of the polydispersity of $l_{c}$ it is more convenient to express the line density in terms of fiber length $L$ such that $\rho_{\mathrm{M}}=\tilde{\rho}_{\mathrm{M}} / L$, where $\tilde{\rho}_{\mathrm{M}}=n_{f} L^{2}$ and $n_{f}$ is the number of rods per unit area [34].

\section{Relationship between model and experimental parameters}

In order to map our model onto experimental parameters, we make three basic assumptions: (1) the filaments are athermal, (2) the filaments behave as rods with a homogenous elasticity, and (3) the network connectivity remains below the isostatic threshold throughout the range of polymerization conditions. Collagen networks, in general, satisfy these assumptions. Collagen fibers are rather thick and thermal fluctuations are therefore unlikely to play a significant role. As for the network connectivity, we have experimentally verified for the concentration range $0.5-4 \mathrm{mg} / \mathrm{ml}$ and at two temperatures $T=30^{\circ} \mathrm{C}$ and $37^{\circ} \mathrm{C}$ that it remains below the isostatic threshold [see Fig. 2(b)].

The most relevant experimental control variable is the total protein concentration $c$. For a given thickness of fibers, the volume fraction $\varphi$ of a network scales linearly with $c$ and using the above assumptions can be simply related to the reduced bending rigidity $\tilde{\kappa}$ as $\varphi \sim \tilde{\kappa} \propto\left(a / l_{0}\right)^{2}[23,33,35,36]$, where $a$ is the fiber thickness. It follows that $K / \varphi$ (or $K / c$ ) in experiments can be directly compared with $\mathcal{K}(\gamma, \tilde{\kappa})$ in simulations.

Our theoretical results depend on the bending rigidity through the parameter $\tilde{\kappa}$ which, as shown above, scales linearly with the protein concentration in experiments. This has an important consequence for the experimental rheology results; the magnitude of modulus and stress as well as the functional dependence of the stiffness on the applied deformation are insensitive to the fibril thickness for a given concentration. This is a direct consequence of the scaling $\tilde{\kappa} \sim \varphi \propto\left(a / l_{0}\right)^{2}$, which remains invariant to changes in fibril thickness for a given total protein concentration. The structure of collagen networks, including fibril thickness, mesh size, homogeneity, and presumably connectivity, depends in detail on concentration and polymerization conditions in nontrivial ways [37,38]. However, under the basic assumptions mentioned above, $\tilde{\kappa}$ remains a constant. 

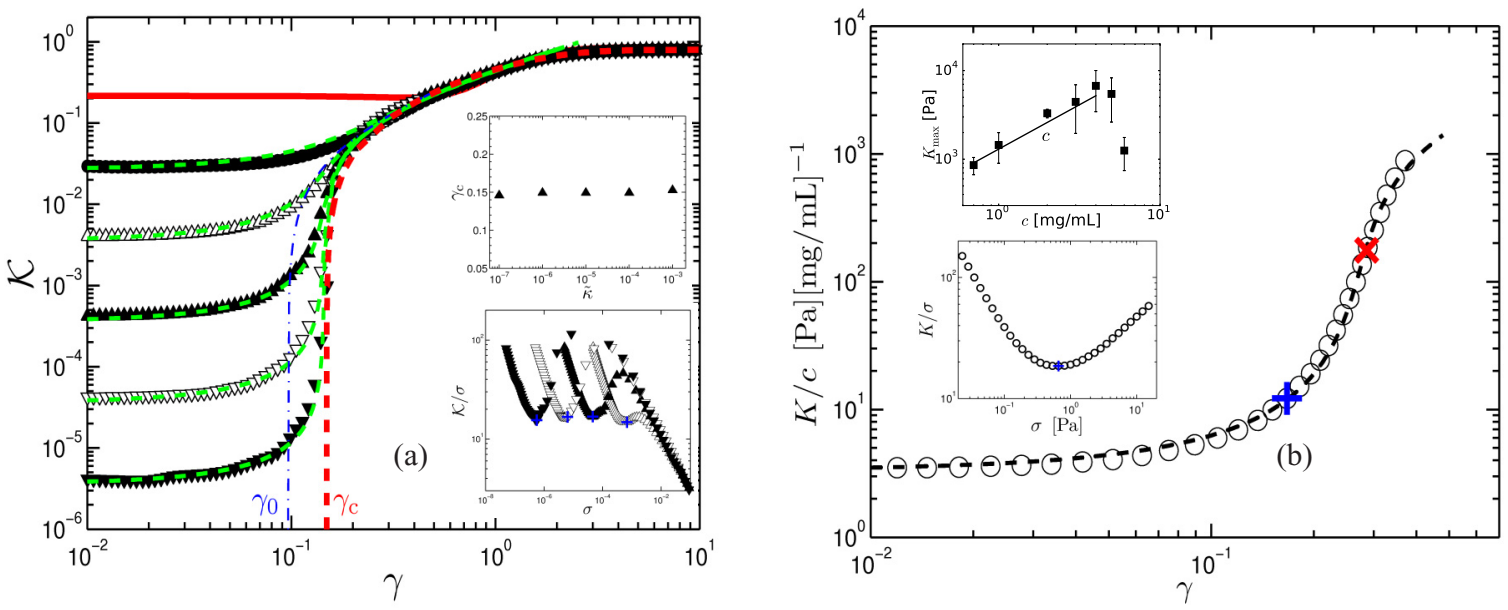

FIG. 1. (a) Shear stiffness versus shear strain curves obtained from a phantom triangular lattice in $2 \mathrm{D}$ with $\langle z\rangle \simeq 3.4$. Different curves are obtained by varying the reduced bending rigidity $\tilde{\kappa}, 10^{-7}(\boldsymbol{\nabla}), 10^{-6}(\nabla), 10^{-5}(\boldsymbol{\Delta}), 10^{-4}(\triangle), 10^{-3}(\bullet)$, and $\infty$ (thick red line). The onset strain for stiffening, $\gamma_{0}$, is shown as the blue dash-dotted line. The red dashed line shows the stiffness when $\tilde{\kappa}=0$. In absence of bending interactions, the stiffness remains zero for $\gamma \leqslant \gamma_{c}$. The green dashed lines through the symbols show the predicted stiffness according to Eq. (9) with $f=0.8 \pm 0.05$ and $\phi=2.1 \pm 0.2$. The top inset shows the strain, identified by the inflection point of the stiffness curves. In the limit of $\tilde{\kappa} \rightarrow 0$, the inflection point approaches the critical strain $\gamma_{c}$ of a central-force subisostatic network. As the inset shows, $\gamma_{c}$ is insensitive to the addition of finite fiber bending rigidity, up to $\tilde{\kappa} \lesssim 10^{-3}$. The bottom inset shows $\mathcal{K} / \sigma$ versus $\sigma$ for different values of $\tilde{\kappa}$. The onset strain for stiffening, $\gamma_{0}$, obtained from the local minimum of $\mathcal{K} / \sigma$ versus $\sigma$, is indicated with a blue cross. (b) Experimentally obtained stiffness versus strain curve for a $1 \mathrm{mg} / \mathrm{ml}$ collagen network $(\bigcirc)$. Since $\mathcal{K}$ in simulations corresponds to $K / c$ in experiments, the experimentally obtained stiffness is normalized to the concentration $c$. The dashed line through the experimental data is fit according to Eq. (9) with the reduced bending rigidity $\tilde{\kappa}=10^{-4}$ and the parameters $f=0.8, \phi=2.3$ obtained from the collapse of stiffness curves obtained from simulations as explained in Sec. III. The critical strain $\gamma_{c}=0.29$, marked with a red cross, is obtained as the inflection point of the stiffness curve. The onset strain for stiffening $\gamma_{0}$ is marked with a blue cross. The top inset shows the experimentally measured $K_{\max }$ versus concentration $c$ for collagen networks, where $K_{\max }$ is the maximum nonlinear modulus before the network ruptures. At large strains, when network stiffness is governed by stretching, $K_{\max }$ is expected to scale with the concentration $c$, shown as the black line. The lower inset shows $K / \sigma$ versus $\sigma$ obtained from the experimental data in (b). The minimum corresponds to the blue cross shown in (b).

\section{STRAIN DRIVEN CRITICALITY}

In Fig. 1(a), we show the network stiffness $\mathcal{K}$ as a function of the applied strain $\gamma$ for different values of $\tilde{\kappa}$. There are three regimes as a function of strain: a linear elastic regime at low strain, a regime of rapid stiffening above a threshold strain $\gamma_{0}$, and a high-strain regime in which the strain dependence is weaker and the elastic modulus becomes independent of $\tilde{\kappa}$. The initial, linear modulus scales linearly with $\tilde{\kappa}$, indicating a bend-dominated response, as has been reported in several prior computational studies $[13,15,18,19,23,33,39]$. The applicability of the computational model for athermal networks such as collagen depends on the following observations. The computational modulus is naturally in units of $\mu \rho$ [Eq. (2)], implying that $G_{0} \equiv K(\gamma=0) \sim \rho \tilde{\kappa} \sim \rho^{2}$. This is consistent with reports of an approximate quadratic concentration dependence on the linear modulus in reconstituted networks of collagen type I [23,33,40,41]. As can be seen in Fig. 1(a) the threshold strain $\gamma_{0}$ for the onset of nonlinear response and eventual breakdown of $\mathcal{K} \sim \tilde{\kappa}$ is a function of network geometry and is insensitive to $\tilde{\kappa}$. As we show, this corresponds experimentally to $\gamma_{0}$ being insensitive to the total protein concentration, which is indeed consistent with several prior experimental studies of reconstituted networks of collagen type I $[23,33,40,41]$. Another important consequence of the computational model is that $\mathcal{K}$ becomes independent of $\tilde{\kappa}$ for large strains, implying that $K \sim \rho$. This is expected when the strains are large enough for the response to be dominated by fiber stretching. In order to test this, we examine $K_{\max }$, the maximum nonlinear modulus of a network before undergoing failure at high stress or strain. Assuming that such failure occurs at sufficiently high strain to enter into the third regime, corresponding to pure stretching, then $\mathcal{K}$ is expected to be insensitive to $\tilde{\kappa}$, corresponding to a linear scaling of $K$ with protein concentration $c$. As can be seen in the inset of Fig. 1(b), the experimentally measured $K_{\max }$ scales linearly with the concentration over a broad range of concentrations, up to approximately $5 \mathrm{mg} / \mathrm{ml}$. Only at the highest concentration studied $(6 \mathrm{mg} / \mathrm{ml})$ do we see a failure of this. While the threshold strain $\gamma_{0}$ corresponds qualitatively to the end of the linear-response regime and onset of nonlinear stiffening, its quantification can be done in various ways. One can, in principle, define $\gamma_{0}$ by the point at which $K$ reaches a fixed multiple (say, twice) of the linear modulus $G_{0}$. Such an approach has been used many times in the prior literature. But, a simple threshold criterion such as this is inherently prone to a systematic shift, in this case toward lower values of the threshold, e.g., due to noisy data. This is expected to systematically underestimate $\gamma_{0}$. We obtain $\gamma_{0}$ as the strain corresponding to the local minimum of $\mathcal{K} / \sigma$ versus $\sigma$ where $\sigma$ is shear stress [see inset of Fig. 1(a)]. We use this same method for both experiments and simulations. In practice, this provides an effective method to determine the onset of 
nonlinear elastic response and the point where the linear relation $\sigma=G_{0} \gamma$ (for which $\mathcal{K} / \sigma$ decreases with $\sigma$ ) breaks down. Importantly, this is less susceptible to systematic error when dealing with noisy data. This method of determining $\gamma_{0}$ is suited to networks with a large enough separation between bend-dominated and stretch-dominated response, for which there is a high (greater than approximately 100-fold) stiffening with strain. In practice, this is the case for networks such as those in Fig. 1(a) with $\tilde{\kappa}<10^{-3}$, which includes the experimentally relevant range. The same method is also used to characterize $\gamma_{0}$ in our experiments, as illustrated by the lower inset of Fig. 1(b). However, in simulations we have confirmed that our conclusions below are otherwise insensitive to the method used to determine $\gamma_{0}$.

When fiber bending costs no energy, i.e., $\tilde{\kappa}=0$, the stiffness $\mathcal{K}$ remains zero for strains $|\gamma| \leqslant \gamma_{c}$. Above $\gamma_{c}, \mathcal{K}$ increases continuously from zero for $\tilde{\kappa}=0$. The critical strain $\gamma_{c}$ is determined by the network architecture, in particular its average connectivity [16]. In a central force subisostatic network, $\gamma_{c}$ can be determined as simply the onset of rigidity strain. When $\tilde{\kappa} \neq 0$, the inflection point of the $\log \mathcal{K}$ versus $\log \gamma$ provides an excellent approximation to the $\gamma_{c}$ in the limit of $\tilde{\kappa} \rightarrow 0$ [Ref. [23] and inset of Fig. 1(a)]. In fact for $\tilde{\kappa} \lesssim 10^{-3}$, the critical strain obtained in this way is practically constant. Moreover, the range of $10^{-5} \lesssim \tilde{\kappa} \lesssim 10^{-3}$ is the experimentally relevant range [24,33]. In particular, the central-force limit is not achievable experimentally. For this reason, $\gamma_{c}$ identified as the inflection point of stiffness curves provides a measure of the critical strain in both simulations and experiments. As shown in Fig. 1(b) and Sec. IV, this approximation is used to determine the $\gamma_{c}$ of the experimentally obtained stiffening curves.

At the isostatic threshold of $\langle z\rangle=2 d$, a central force network is marginally stable with $\gamma_{c}=0$. In Fig. 2(a), we show a schematic of the phase diagram in $\gamma-z$ plane. For a given average connectivity below the isostatic threshold, increasing the deformation beyond $\gamma_{c}$ causes a phase transition from floppy to rigid phase. The continuous curve $\gamma_{c}(z)$ marks the boundary between the floppy and rigid states of subisostatic networks. In Fig. 2(b) we show the computationally obtained $\gamma_{c}$ versus the average connectivity in the network when subjected to simple shear deformation. For each value of $\langle z\rangle$, we considered a minimum of eight network configurations to obtain the critical strain. The critical strains for both 2D and 3D networks are in quantitative agreement as long as the connectivity is sufficiently below the $2 \mathrm{D}$ isostatic point $\langle z\rangle=4$. The shaded region in Fig. 2(b) spans the connectivities relevant for collagen networks. Also shown are two values of $\gamma_{c}$ of $4 \mathrm{mg} / \mathrm{ml}$ collagen networks for two temperatures. The critical strain is in quantitative agreement with the model for $T=30^{\circ} \mathrm{C}$. The apparent disagreement for $T=37^{\circ} \mathrm{C}$ is probably due to the uncertainty associated with determination of $\langle z\rangle$ in the experiments. It is possible that due to finite resolution in experiments, the connectivity at some of the nodes is measured as 4 due to overlapping collagen fibers. This would lead to an overestimation of $\langle z\rangle$ and can thus account for the disagreement between theory and experiments at $T=37^{\circ} \mathrm{C}$.

In Fig. 3, the network stiffness is shown for several values of $\tilde{\kappa}$ in the vicinity of the critical strain. The continuous nature
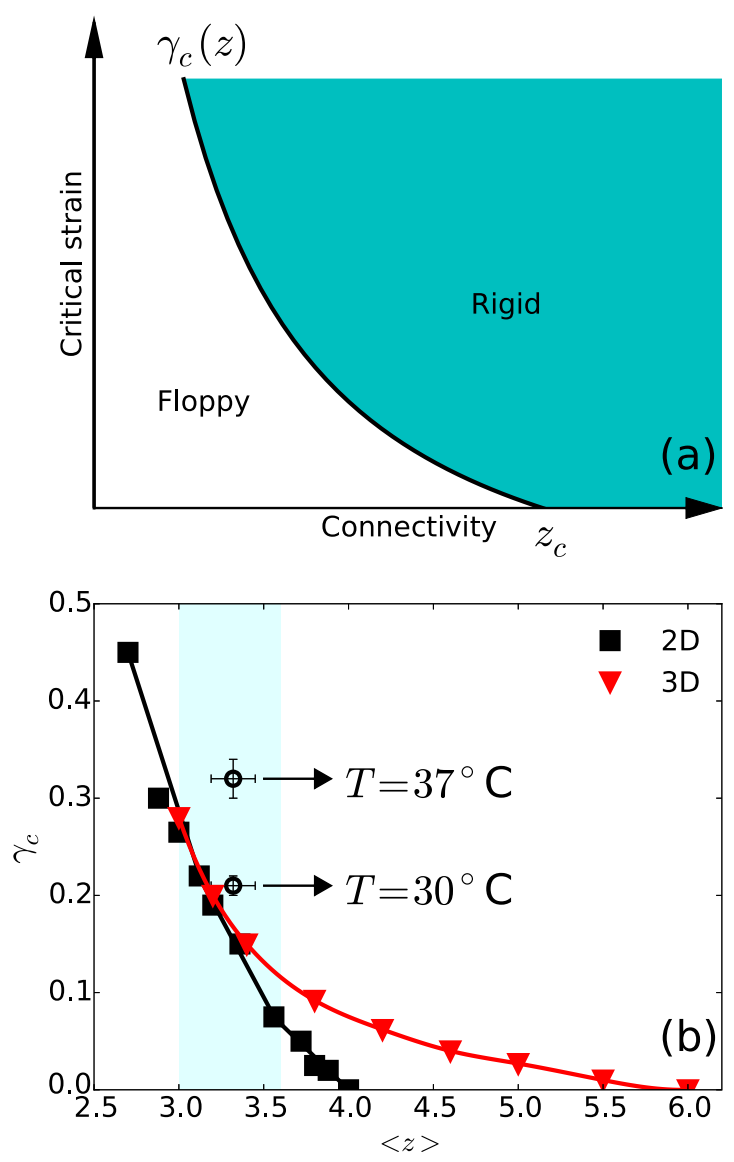

FIG. 2. (a) Schematic diagram of the phase behavior of disordered fibrous networks. The curve $\gamma_{c}(z)$ is the boundary between floppy and rigid states. (b) $\gamma_{c}$ versus average connectivity for phantom triangular networks in 2D and FCC lattice-based 3D networks. The critical strain decreases with increasing connectivity and approaches zero at the isostatic threshold $\langle z\rangle=2 d$. The shaded region spans connectivities in the range 3.0-3.6. The two open symbols correspond to $\gamma_{c}$ of collagen networks prepared at $4 \mathrm{mg} / \mathrm{ml}$ for two different polymerization temperatures. The symbols show average of three samples and error bars represent standard deviations. Per sample at least 100 junctions were measured to determine $\langle z\rangle$.

of the transition from floppy to rigid states is evident in the critical scaling of the network stiffness $\mathcal{K} \sim|\Delta \gamma|^{f}$ where $\Delta \gamma=\gamma-\gamma_{c} \geqslant 0$ and $f$ is a critical exponent. As shown in Fig. 3, the power-law scaling of stiffness is apparent only in the limit of $\tilde{\kappa} \rightarrow 0$. Extracting $f$ as the limiting slope of $\mathcal{K}$ versus $\Delta \gamma$ provides an independent method of obtaining this critical exponent. The same exponent can be obtained by scaling analysis as described in Sec. III A. Power-law scaling of the order parameter, $\mathcal{K}$ (or $K$ ) in our case, is a hallmark signature of critical phenomena. In fact, the strain-driven phase transition is strictly defined in the thermodynamic limit of $W \rightarrow \infty$ and $\tilde{\kappa}=0$ at which the interactions within the network are purely central force interactions. In a finite-size subisostatic network with $\tilde{\kappa}=0$, the stiffness remains finite at the critical strain and scales as $W^{-f / v}$ [23] where $v$ is the exponent associated with divergence of correlation length. Upon addition of a field such as fiber bending, the network becomes stable for $\gamma<\gamma_{c}$ with the stiffness $\mathcal{K} \propto \tilde{\kappa}$. 


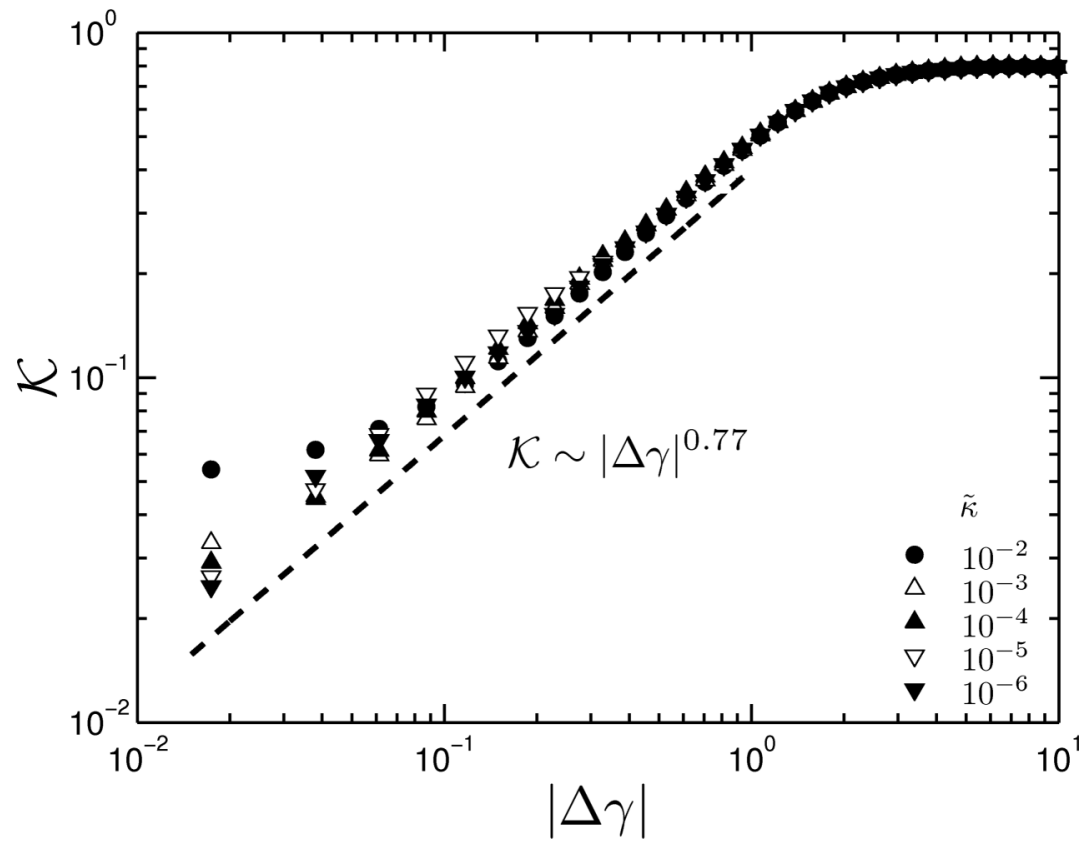

FIG. 3. Shear stiffness $\mathcal{K}$ versus $\Delta \gamma=\gamma-\gamma_{c}$ for different $\tilde{\kappa}$ obtained from simulations on a phantom triangular network in $2 \mathrm{D}$ with $\langle z\rangle \simeq 3$.4. In the limit of $\tilde{\kappa} \rightarrow 0$, the stiffness $K$ increases as a power-law in $\Delta \gamma$ with the critical exponent $f$, which for the given network is $\simeq 0.77$.
In absence of bending interactions, the phase behavior characterized by the continuous transition of the order parameter $\mathcal{K}$ is reminiscent of the ferromagnetic phase transition. Magnetic materials are characterized by a Curie temperature $T_{c}$ such that for $T$ above $T_{c}$, the material is paramagnetic. On lowering the temperature $T$ below $T_{c}$, there is spontaneous magnetization $M$ of the material which increases continuously from zero as $M \propto|\Delta T|^{\beta}$ where $\Delta T=T-T_{c}<0$ and $\beta$ is the critical exponent. Above the Curie temperature, the paramagnetic phase is characterized by a zero magnetization. However, in presence of a finite magnetic field $H$, there is a net magnetization in the paramagnetic phase with $M \propto H$. It is an intriguing analogy that by mapping $\tilde{\kappa}$ to external field $H$ and $\gamma$ to the temperature $T$, one can study the transition from floppy to rigid states the same way as in a ferromagnet as further elaborated in Sec. IV.

\section{A. Crossover for finite $\tilde{\kappa}$}

The power law scaling of $\mathcal{K}$ with $\Delta \gamma$ is a hallmark signature of criticality and is strictly observed only when $\tilde{\kappa}=0$. It is obvious that in this regime, the modulus is entirely governed by stretching of fibers. For any finite $\tilde{\kappa}$, a subisostatic network is stable for $\Delta \gamma<0$. In fact, for sufficiently small $\tilde{\kappa}$, the linear modulus of a subisostatic network is bending governed leading to $\mathcal{K} \sim \tilde{\kappa}$ for $\gamma<\gamma_{c}[13,15,18,19,23,33]$. Analogous to ferromagnetism, in presence of finite auxiliary field $\tilde{\kappa}$, the network undergoes a strain driven crossover from the bend dominated regime $\Delta \gamma<0$ to the stretch dominated regime $\Delta \gamma>0$. These two regimes can be summarized by the scaling form

$$
\mathcal{K} \propto|\Delta \gamma|^{f} \mathcal{G}_{ \pm}\left(\frac{\tilde{\kappa}}{|\Delta \gamma|^{\phi}}\right)
$$

where $\mathcal{G}_{ \pm}$is a scaling function with the positive and negative branches corresponding to $\Delta \gamma>0$ and $\Delta \gamma<0$, respectively. This scaling is analogous to that for the conductivity of random resistor networks and fiber networks as a function of connectivity $[15,42]$. In Fig. 4, we test this by plotting $\mathcal{K}|\Delta \gamma|^{-f}$ versus $\tilde{\kappa}|\Delta \gamma|^{-\phi}$, according to Eq. (3). For $x \ll 1$, $\mathcal{G}_{+}(x)$ is approximately constant and $\mathcal{G}_{-}(x) \propto x$. That $\mathcal{G}_{+}(x)$ is approximately constant for $x \ll 1$ captures the critical scaling of $K$ as $\mathcal{K} \sim|\Delta \gamma|^{f}$. The scaling $\mathcal{G}_{-}(x) \propto x$ captures the bend-dominated linear modulus where the linear modulus scales as $\mathcal{K} \sim \tilde{\kappa}$. Since $K$ must be finite at $\Delta \gamma=0$, we also expect $\mathcal{K} \sim \kappa^{f / \phi} \mu^{1-f / \phi}$, consistent with Eq. (3). We show in Fig. 4 the data obtained from phantom triangular networks in 2D [same as in Fig. 1(a)] and FCC-based 3D lattices collapsed according to Eq. (3). Interestingly, the data collapse with the same exponents $f \simeq 0.8$ and $\phi \simeq 2.1$. The average connectivity for the two different networks is chosen to be $\simeq 3.4$. Data from Mikado networks with the same average

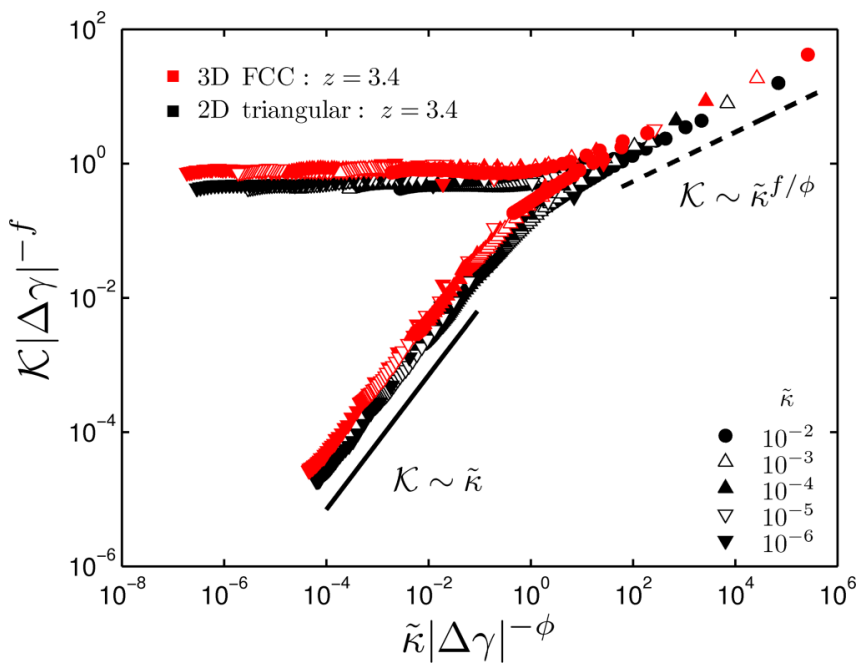

FIG. 4. (a) Collapse of shear stiffness versus shear strain curves of Fig. 1(a) according to Eq. (3). Simulation data from 3D network with same connectivity as in $2 \mathrm{D}$ of $\langle z\rangle \simeq 3.4$ collapse with the same critical exponents $f=0.8$ and $\phi=2.1$. 

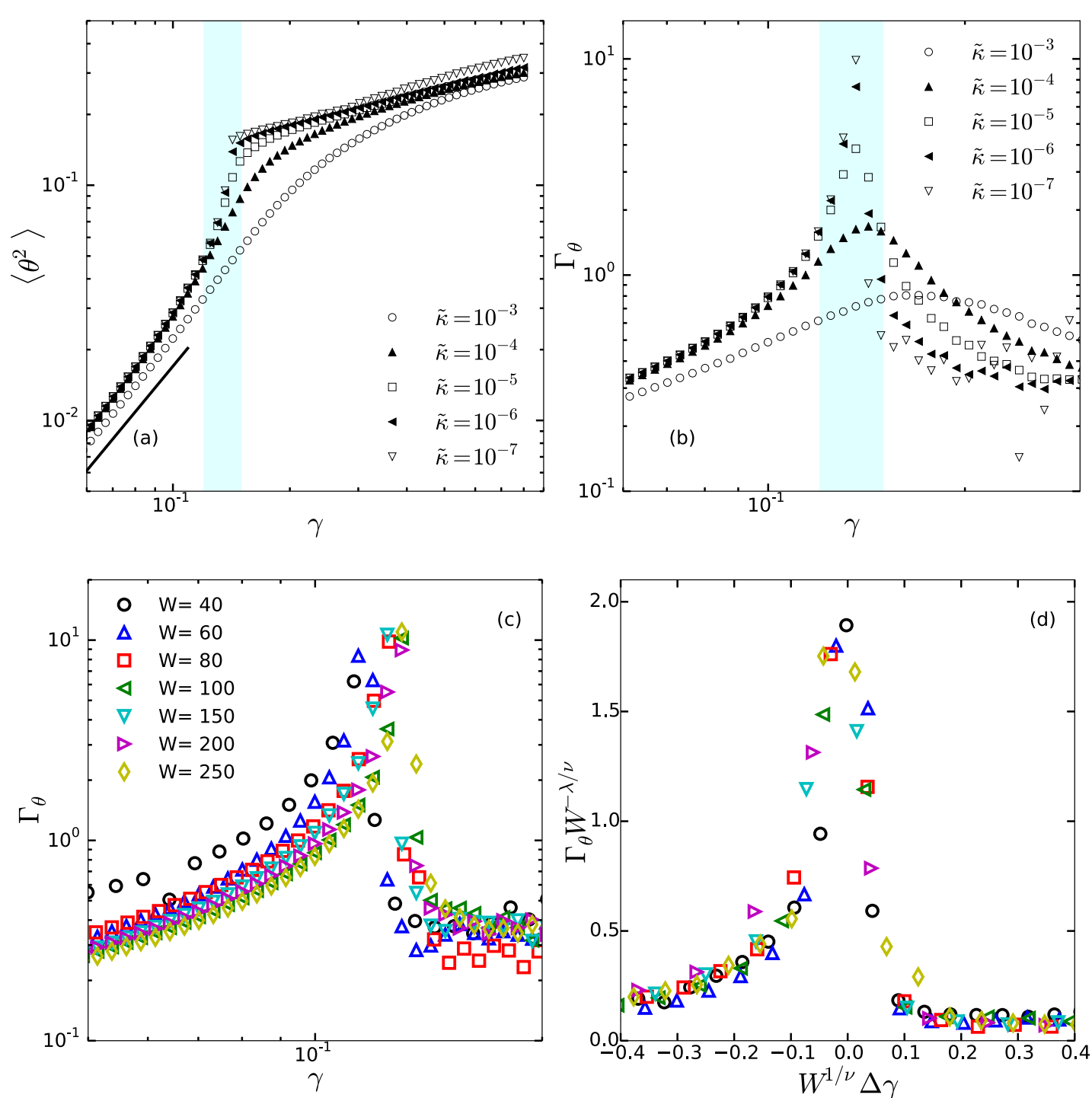

FIG. 5. Divergent fluctuations at the critical strain. (a) Average bending angle $\left\langle\theta^{2}\right\rangle$ obtained from simulations on a phantom triangular network in $2 \mathrm{D}$ with $\langle z\rangle \simeq 3.4$ for different values of $\tilde{\kappa}$ (see legend). The network size is $W^{2}=250^{2}$. The thick black line indicates the expected small-strain $\gamma^{2}$ scaling. $\left\langle\theta^{2}\right\rangle$ increases monotonically with $\gamma$. The shaded region is approximately in the range $\gamma_{c}-\gamma_{0}$. In this range, the rate of increase of $\left\langle\theta^{2}\right\rangle$ is strongly dependent on $\tilde{\kappa}$. (b) $\Gamma_{\theta}(\gamma)$ obtained as the derivative of data in (a) with respect to $\gamma$. In the limit of $\tilde{\kappa} \rightarrow 0$, $\Gamma_{\theta}$ diverges at $\gamma=\gamma_{c}$. (c) $\Gamma_{\theta}$ versus $\gamma$ for different system sizes (see legend). The bending rigidity is $\tilde{\kappa}=10^{-7}$. (d) Collapse of data in (c) according to Eq. (5) with $\lambda=0.6 \pm 0.1$ and $v=2.0 \pm 0.1$.

connectivity $\langle z\rangle$ as in lattice-based networks can be collapsed with the same critical exponents [23]. In fact, as we show in Sec. VI, the exponents appear to be independent of the spatial dimensionality and are primarily determined by the average connectivity.

Mapping protein concentration to $\tilde{\kappa}$ as described in Sec. II A allows us to obtain an analogous scaling relation applicable to experimental data. Since computationally one obtains $\mathcal{K}$ one must create the analogous quantity in experiments by scaling the measured modulus with concentration, i.e., $K / c$. On substituting $c$ for $\tilde{\kappa}$ and $K / c$ for $\mathcal{K}$ in Eq. (3), we obtain the scaling function to collapse the experimental data as shown by us in Ref. [23].

The scaling function $\mathcal{G}_{ \pm}$, with $f$ and $\phi$ as input parameters, describes the stiffening curves over the entire elastic regime for any concentration (or $\tilde{\kappa}$ in simulations). One can obtain an analytical $\mathcal{G}_{ \pm}$(approximately) exploiting the analogy of nonlinear mechanics to ferromagnetism as we show in Sec. IV.

\section{B. Divergent fluctuations}

In a thermal critical phenomenon, there are divergent fluctuations in the order parameter at the critical point. In the athermal network under consideration in this study, there are no divergent fluctuations in the macroscopic $\mathcal{K}$. One can, however, measure fluctuations by considering the deviation of the strain field within the network from the expected affine field $[32,43]$. Under affine deformation, filaments are either stretched or compressed. Deviations from the affine deformation induce bending on filaments which can be considered as a measure of fluctuations. These fluctuations are suppressed by a finite field such as $\tilde{\kappa}$. In Fig. 5(a), we plot the bending angle $\theta_{i j k}$ 

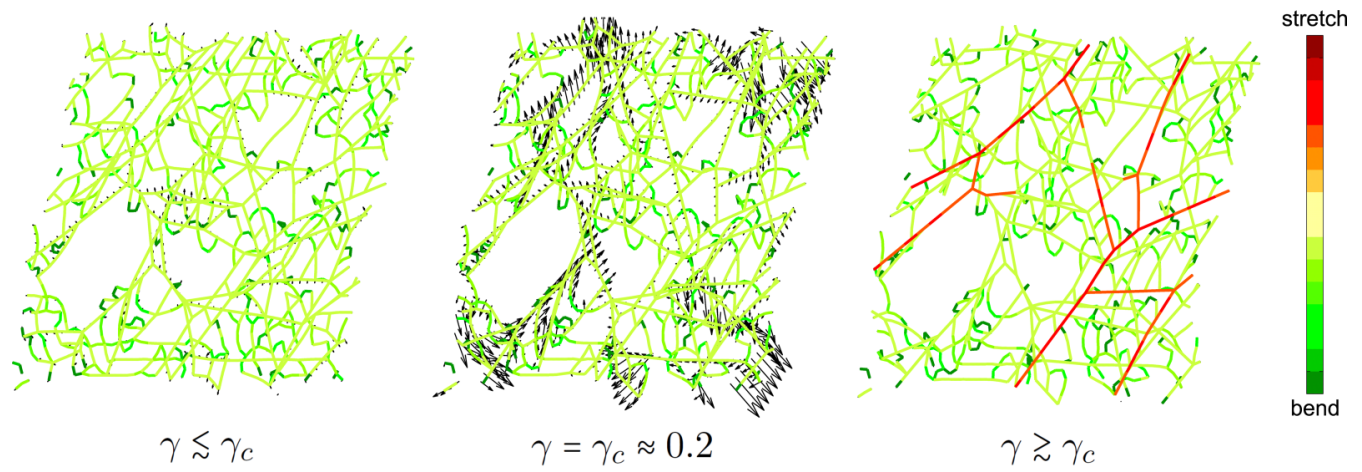

FIG. 6. Nonaffine displacements in a $2 \mathrm{D}$ phantom triangular network with $\langle z\rangle \simeq 3.4$ and $\tilde{\kappa}=10^{-6}$ are shown as the network is deformed through the critical strain $\gamma_{c}$. The arrows indicate the deviation of a node from the imposed deformation. The magnitude of the vectorial displacements is largest at the critical strain. The color bar on the right indicates the elastic energy in bending (green) or stretching (red) form. The network size is $W=20 l_{0}$.

averaged over the entire network for different values of $\tilde{\kappa}$. The triplet $\{i, j, k\}$ corresponds to three consecutive crosslinks labeled as $i, j$, and $k$ and the average implies summing over all the triplets in the network. As can be seen in Fig. 5(a), the average bending angle increases with the applied deformation. For small strains, the increase is quadratic in $\gamma$ as expected in the linear regime. At large strains, the average bending angle increases very slowly with the applied deformation. In the intermediate strain range, shown as the shaded region, the rate of increase of average bending angle depends strongly on $\tilde{\kappa}$. We define $\Gamma_{\theta}$ as the rate of change of the average bending angle with the applied strain:

$$
\Gamma_{\theta}(\gamma)=\frac{\partial\left\langle\theta_{i j k}^{2}\right\rangle}{\partial \gamma} .
$$

In Fig. 5(b), we plot $\Gamma_{\theta}$ as a function of $\gamma$ for different values of $\tilde{\kappa}$. These results are obtained from simulations on a phantom triangular network in $2 \mathrm{D}$ with $\langle z\rangle \simeq 3.4$. The maximum of $\Gamma_{\theta}$ shifts to the left in $\gamma$ with decreasing bending rigidity. In the limit of $\tilde{\kappa}=0$, the peak height is maximum for a given network size and it is located at the critical strain $\gamma=\gamma_{c}$.

The quantity $\Gamma_{\theta}$ is expected to diverge in the thermodynamic limit at $\gamma=\gamma_{c}$ for $\tilde{\kappa}=0$. In Fig. 5(c), we show $\Gamma_{\theta}$ for different system sizes $W$. These curves are obtained for a fixed small $\tilde{\kappa}=10^{-7}$. If $\Gamma_{\theta}$ diverges as $\left|\gamma-\gamma_{c}\right|^{-\lambda}$ in the thermodynamic limit $W \rightarrow \infty$, then the following scaling relation must capture the scaling behavior of $\Gamma_{\theta}$ for finite $W$ :

$$
\Gamma_{\theta} \propto W^{\lambda / v} \mathcal{H}\left(W^{1 / \nu} \Delta \gamma\right),
$$

where $v$ is the exponent associated with the divergence of correlation length [23], $\Delta \gamma=\gamma-\gamma_{c}$ is the distance from the critical strain, and $\mathcal{H}(x)$ is a scaling function. We show in Fig. 5(d), the collapse of data in Fig. 5(c) according to Eq. (5) with the exponents $\lambda=0.6 \pm 0.1$ and $v=2.0 \pm 0.1$. With these exponents, the peak height of $\Gamma_{\theta}$ is expected to scale as $W^{\lambda / v} \sim W^{0.3}$. It follows that due to the weak system size dependence, a clear demonstration of $W^{\lambda / v}$ scaling of the peak height requires much larger system sizes than those studied in this work. Nevertheless, the collapse in Fig. 5(d) provides convincing evidence for $\Gamma_{\theta}$ as an appropriate measure of fluctuations in fibrous networks.
Another measure of fluctuations is the differential nonaffinity, which measures the strain fluctuations within the network. Given the displacement field $\mathbf{u}$ and the affine displacement field $\mathbf{u}^{\mathrm{A}}$ of the network, the nonaffine fluctuations can be quantified as [16]

$$
\delta \Gamma(\gamma)=\frac{\left\langle\left\|\delta \mathbf{u}^{\mathrm{NA}}\right\|^{2}\right\rangle}{l^{2} d \gamma^{2}},
$$

where $\delta \Gamma(\gamma)$ is referred to as differential nonaffinity, $\delta \mathbf{u}^{\mathrm{NA}}=\mathbf{u}-\mathbf{u}^{\mathrm{A}}$ is the differential non-affine displacement of a crosslink to an imposed strain $d \gamma, l$ is the typical network mesh size, and the angular brackets represent a network average. In Ref. [23], we showed that $\delta \Gamma(\gamma)$ exhibits a peak at $\gamma=\gamma_{c}$, the height of which increases with decreasing $\tilde{\kappa}$. In Fig. 6, we show the differential nonaffine displacements $\delta \mathbf{u}^{\mathrm{NA}}$ superimposed on network nodes in the neighborhood of $\gamma_{c}$. The magnitude of nonaffine displacements is largest at the critical strain. It follows that the network is at its most susceptible mechanical state at $\gamma=\gamma_{c}$ requiring large scale internal rearrangements in response to an infinitesimal external deformation. The nature of deformation within the network changes dramatically when the applied deformation increases through $\gamma=\gamma_{c}$. Whereas the network deforms primarily through bending modes for $\gamma \leqslant \gamma_{c}$, stretching becomes the dominant deformation mode for $\gamma>\gamma_{c}$.

Finite-size scaling analysis of the order parameter $\mathcal{K}$ reveals underlying divergence of the correlation length as shown in Ref. [23]. The diverging correlation length, together with divergent fluctuations and the continuously evolving order parameter constitute evidence in favor of a second-order-type strain-driven phase transition in disordered networks.

\section{EQUATION FOR THE CROSSOVER FUNCTION}

The scaling ansatz and function $\mathcal{G}_{ \pm}(x)$ in Eq. (3) can account well for the nonlinear mechanics of our model networks for any $\tilde{\kappa}$ and $\gamma$. We can obtain an analytical approximation for $\mathcal{G}_{ \pm}(x)$ in a way analogous to the approach for ferromagnetism $[44,45]$. In a way similar to the equation of state relating magnetic field $H$ to magnetization $M$, we postulate the following mean-field equation of state for bending stiffness 
$\tilde{\kappa}$ and as a series in the shear modulus $\mathcal{K}$ [46]:

$$
\tilde{\kappa} \sim b \mathcal{K}+c \mathcal{K}^{2}
$$

where $b \sim \Delta \gamma$ for a transition controlled by strain. Here, in contrast with the order parameter $M$ for ferromagnetism, symmetry does not forbid a quadratic term in this equation of state [46]. After a minor change in normalization, this can be rewritten as

$$
\frac{\tilde{\kappa}}{|\Delta \gamma|^{2}} \sim \frac{\mathcal{K}}{|\Delta \gamma|}\left(\mp 1+\frac{\mathcal{K}}{|\Delta \gamma|}\right),
$$

where the upper "-_" refers to $\gamma>\gamma_{c}$ and the lower " + " refers to $\gamma<\gamma_{c}$. This yields $\mathcal{K} \sim|\Delta \gamma|$ for small $\Delta \gamma>0$ and $\tilde{\kappa}=0$, while $\mathcal{K} \sim \tilde{\kappa}$ for $\Delta \gamma<0$ and small $\tilde{\kappa}>0$. As shown above, our results deviate from the mean-field behavior, $\mathcal{K} \sim|\Delta \gamma|^{f}$, where $f=1$. We find $f \simeq 0.8$.

As is done for ferromagnetism, the equation of state above can be written in a form that can account for non-mean-field exponents, while remaining nonsingular except at the critical point $(\Delta \gamma=\tilde{\kappa}=0)$. We introduce potentially noninteger exponents $f$ and $\phi$, where

$$
\frac{\tilde{\kappa}}{|\Delta \gamma|^{\phi}} \sim \frac{\mathcal{K}}{|\Delta \gamma|^{f}}\left(\mp 1+\frac{\mathcal{K}^{1 / f}}{|\Delta \gamma|}\right)^{(\phi-f)} .
$$

For $\Delta \gamma=0$, this scaling relation corresponds to $\mathcal{K} \sim \tilde{\kappa}^{f / \phi}$ at the critical point. Again, the mean-field values of the exponents are $f=1$ and $\phi=2$.

Equation (9) can be used to calculate $\mathcal{K}$ for any $\gamma$. The input parameters are $\tilde{\kappa}, f, \phi$, and $\gamma_{c}$. The critical strain $\gamma_{c}$ can be independently determined from a network with only central-force interactions. The critical exponents are obtained from the data collapse using Eq. (3). In Fig. 1(a), we use Eq. (9) to obtain $\mathcal{K}$ as a function of $\gamma$ for different $\tilde{\kappa}$. The stiffening curves calculated using Eq. (9) are shown together with the numerically obtained curves. Clearly, Eq. (9) can accurately predict the nonlinear stiffening curves.

Equation (9) can accurately capture the experimentally obtained stiffening curves of collagen networks [23]. However, the fitting procedure, when applied to experiments needs to be slightly modified. The fitting to experimental data is done in the following way. We first focus on the linear regime. In the linear regime, we know from simulations that the modulus (in units of $\rho \mu$ ) scales linearly with $\tilde{\kappa}$, which itself scales as $\tilde{\kappa} \sim \rho$ giving rise to a $c^{2}$ (or $\rho^{2}$ ) dependence of the linear modulus where $c$ is the protein concentration. However, as shown in the inset of Fig. 1(b), the linear modulus obtained experimentally from reconstituted collagen networks exhibits $K \sim c^{2+\delta}$ scaling. It is plausible that the deviation from the $c^{2}$ scaling is simply a consequence of experimental uncertainties. However, as shown in Ref. [23], the deviation from $c^{2}$ scaling is probably due to the weak dependence of $\gamma_{c}$ on the concentration of collagen in experiments. In this section, we simply rescale the experimental $K$ by $c^{1+\delta}$ such that rescaled modulus scales as $K / c^{1+\delta} \sim c \sim \tilde{\kappa}$. Next, we obtain the individual critical strains, $\gamma_{c}$, for each of the concentrations as the inflection point of the $\log K$ versus $\log \gamma$ curve. We then consider the experimental data (rescaled by $c^{1+\delta}$ ) for each concentration along with its $\gamma_{c}$ and fit the entire curve to Eq. (9) with $\tilde{\kappa}$ as the only free parameter. Here we show the result of the fitting

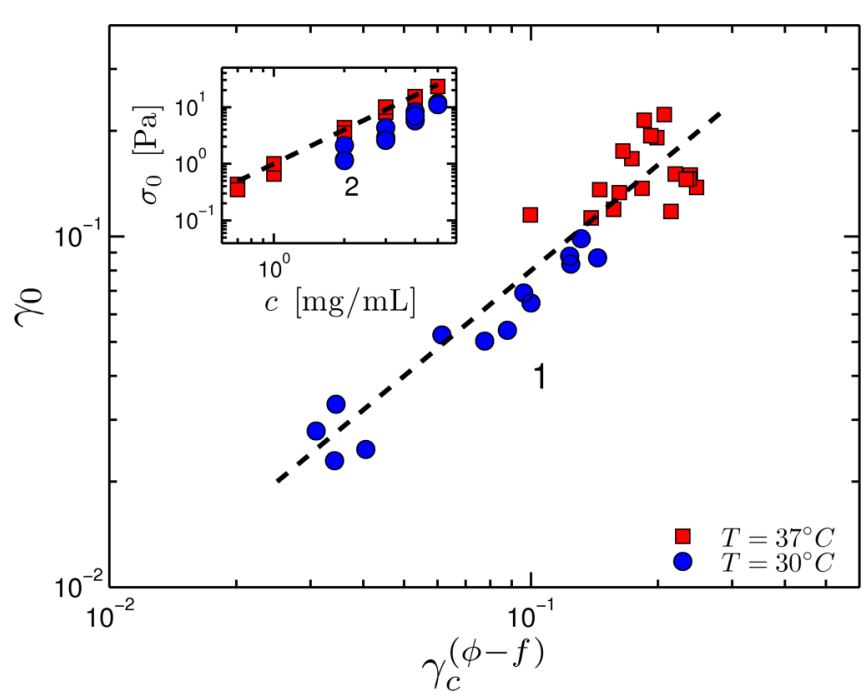

FIG. 7. The onset strain for stiffening scales as $\gamma_{0} \sim \gamma_{c}^{(\phi-f)}$. The critical exponents are $\phi=2.1$ and $f=0.8$. The experimental data are taken from collagen networks prepared at temperatures, $T=30^{\circ} \mathrm{C}$ ( $)$ and $37^{\circ} \mathrm{C}(\square)$. This scaling is a direct consequence of the measured $c^{2}$ scaling of the shear stress at $\gamma_{0}$ as shown in the inset.

for a $1 \mathrm{mg} / \mathrm{ml}$ collagen network in Fig. 1(b) superimposed on the experimental data. We have reported the full set of experimental curves over a wide range of concentrations of collagen along with the fitting in Ref. [23].

\section{RELATION BETWEEN $\gamma_{0}$ AND $\gamma_{c}$}

In a recent study, we showed that the onset of stiffening strain $\gamma_{0}$ is practically independent of the concentration of collagen [33]. The invariance of the geometrical structure of the network with concentration, in particular of the average connectivity in the network, was suggested as the underlying reason for the independence. Both $\gamma_{0}$ and $\gamma_{c}$ are fully determined by the connectivity and geometry of the network and hence are well-defined quantities for any subisostatic network with finite $\tilde{\kappa}$. It is therefore expected that a general relation exists between $\gamma_{0}$ and $\gamma_{c}$.

An expression for $\gamma_{0}$, based on geometrical arguments has been derived in Ref. [17]. We can obtain an expression for $\gamma_{c}$ in terms of $\gamma_{0}$ and critical exponents in the following way. Using Eq. (3), the linear modulus $G_{0}$ can be written as $G_{0} \equiv K(\gamma=0) \sim c^{2} \gamma_{c}^{f-\phi}$. It follows that the stress at the onset of stiffening should scale as $\sigma_{0}=G_{0} \gamma_{0} \sim c^{2} \gamma_{c}^{f-\phi} \gamma_{0}$. The experimentally obtained $\sigma_{0}$ versus concentration is shown in the inset of Fig. 7. The data are taken from collagen networks prepared at temperatures, $T=30^{\circ} \mathrm{C}$ and $37^{\circ} \mathrm{C}$. As can be seen in Fig. 7, $\sigma_{0}$ scales quadratically with the concentration implying that

$$
\gamma_{0} \sim \gamma_{c}^{(\phi-f)}
$$

This scaling relation accurately describes the relation between $\gamma_{0}$ and $\gamma_{c}$ as shown in Fig. 7 with $\phi=2.1$ and $f=0.8$. However, unlike $\gamma_{0}$, which can be determined analytically, determination of $\gamma_{c}$ from Eq. (10) requires the knowledge of the critical exponents which, at present, are only obtained from scaling analysis of stiffening data. The critical strain $\gamma_{c}$ 


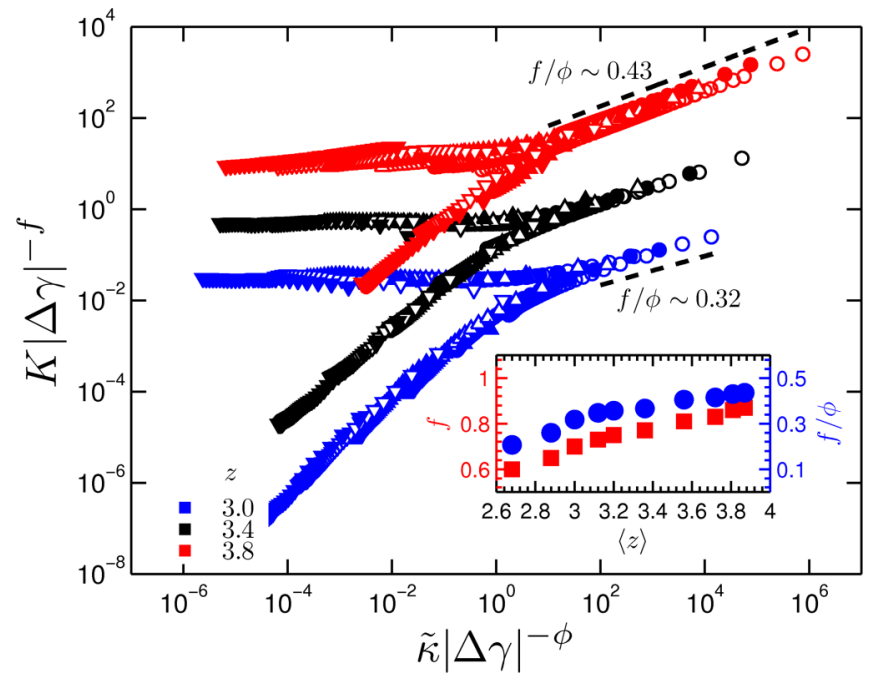

FIG. 8. Shear stiffness versus shear strain curves collapsed according to the Eq. (3) for phantom triangular networks in 2D prepared at different connectivities (see legend). The red and the blue data sets have been shifted by a decade up and down, respectively, for better visualization. The exponent $f$ changes significantly with $\langle z\rangle$. With $\phi$ showing practically no dependence on the connectivity, the ratio $f / \phi$ increases with the connectivity as shown in the inset.

as shown in Fig. 7 corresponds to networks prepared over a range of total protein concentration (see inset of Fig. 7). The variation of $\gamma_{c}$ with concentration is not inconsistent with the fact that $\gamma_{c}$ should be fully determined by the connectivity and geometry of the network. As we pointed out in Ref. [23], the average connectivity in the network may weakly depend on the concentration giving rise to an indirect concentration dependence of $\gamma_{c}$. It is important to note that the above arguments are valid only when the average connectivity in the network depends weakly on the concentration. This requirement is based on the observation, as shown in the next section, that the critical exponents evolve with the average connectivity in the network. Using a unique set of values for $\phi$ and $f$ in Eq. (10) requires that these two exponents are practically constant over the entire range of collagen concentrations.

\section{CRITICAL EXPONENTS AND CONNECTIVITY}

Strikingly, the critical exponents obtained by collapsing both simulation data of $2 \mathrm{D}$ and $3 \mathrm{D}$ fibrous networks and experimental data for collagen networks are identical [23] as long as the average network connectivity is the same. The exponents are apparently independent of the spatial dimensionality. This is in contrast to both thermal and athermal critical phenomena where the critical exponents depend on the spatial dimensionality [45,47]. In fact, the critical exponents evolve with the average connectivity in the network. In Fig. 8, we show the nonlinear stiffness data collapsed according to Eq. (3) for 2D triangular lattice-based networks prepared at different connectivities. The inset of Fig. 8 shows a plot of $f$ and $f / \phi$ versus the average connectivity for both $2 \mathrm{D}$ and $3 \mathrm{D}$ lattice-based networks. It is clear that $f$ increases with the average connectivity in the network, whereas $\phi$ remains practically constant. The evolution of critical exponents with the connectivity has been also observed in branched networks modeled as diluted honeycomb structures [48].

The continuous variation of critical exponents is similar to the behavior of Ashkin-Teller and 8-vertex models, which exhibit continuously varying critical exponents along a critical line [49-51]. Such a variation in the critical exponents has been experimentally observed in certain quantum phase transitions [52,53]. In Ref. [48], we presented a hypothesis that the apparent variation of the critical exponents could correspond to a crossover between critical exponents in the pure and disordered limits where the pure limit corresponds to an undiluted and undistorted perfect lattice based network. At present it remains unclear whether the variation can be attributed to a crossover behavior. However, based on previous simulations [16] an interesting experimental verification of varying exponents could be to isotropically compress a subisostatic random network, since this would reduce the number of constraints while leaving the connectivity the same.

\section{DISCUSSION AND CONCLUSIONS}

In this study, we focus on the mechanical critical behavior in fiber networks. The networks considered are athermal, disordered, and are by construction, subisostatic. The criticality is driven by the applied global deformation and is the fundamental mechanism of the nonlinear mechanics of such networks. Unlike the isostatic connectivity threshold that depends on the precise balance of the number of constraints to the degrees of freedom, any generic subisostatic network exhibits critical behavior when subjected to an external deformation. The criticality is evident in the neighborhood of a strain that is determined by the network architecture.

One of the hallmark features of critical phenomena is the power-law scaling of the order parameter in the vicinity of the critical point. We show that the stiffness of subisostatic networks with central-force interactions scales as a powerlaw, $\mathcal{K} \sim|\Delta \gamma|^{f}$, where $\Delta \gamma=\gamma-\gamma_{c} \geqslant 0$ is the distance measured from the critical strain and $f$ is a critical exponent. Additional interactions such as resistance to bending stabilize subisostatic networks in the subcritical regime $\Delta \gamma<0$ such that for $\gamma \ll \gamma_{c}, \mathcal{K} \sim \tilde{\kappa}$ where $\tilde{\kappa}$ is the bending rigidity. From the perspective of a critical phenomenon, finite bending rigidity can be considered as an auxiliary field that suppresses the strain-driven criticality. For $\tilde{\kappa}>0$ the stiffness at the critical strain is finite and depends in a power-law fashion on the strength of bending and stretching interactions. Drawing analogy with the ferromagnetic phase transition, where $H$, the applied magnetic field is the auxiliary field, we capture the crossover of stiffness from bend-dominated to stretch-dominated regimes in terms of a universal scaling function.

Another important signature of criticality besides the power-law scaling of the order parameter is the divergence of fluctuations in the order parameter at the critical point. In athermal subisostatic networks, the order parameter $\mathcal{K}$ is zero at the critical strain and exhibits no fluctuations. However, on considering the deviation of the strain field within the network from the globally imposed affine field, 
one can create measures for fluctuations. We construct one such measure: the strain-derivative of average bending-angle in the network and using finite-size scaling demonstrate its divergence in the thermodynamic limit. Recently $\mathrm{Xu}$ et al. have developed an image analysis software SOAX, which can accurately track fibers in 3D [54]. It is an interesting idea to use SOAX together with confocal shear cell rheology [55] to experimentally measure the average bending angle in reconstituted biopolymer networks.

The analogy with the ferromagnetic phase transition guides us in writing an approximate equation for the scaling function that captures the crossover of stiffness from bend-dominated to stretch-dominated regimes. We demonstrate that the derived equation is highly accurate in describing the entire nonlinear stiffness versus strain curves for any bending rigidity. Since concentration in experiments can be mapped to the reduced bending rigidity in our network model, the equation for the crossover function can equivalently describe the stiffness versus strain curves for any concentration of the protein in the experiments. We show that the equation accurately describes the stiffness of collagen networks with a single fit parameter. The excellent agreement of model predictions with the experiments provides strong evidence for criticality as the underlying mechanism of the well-known nonlinear mechanics of athermal fibrous networks such as collagen [23,33,40,41] and bundled actin [56-58].
A surprising observation is that under simple shear, the critical exponents $f$ and $\phi$ appear to be independent of the spatial dimensionality. This is a highly intriguing and also puzzling observation. The critical exponents, as is known from the theory of critical phenomena, depend on the spatial dimensionality. However, the exponents are not constant as they change with the average connectivity in the network. The variation of critical exponents along a critical line is similar to the Ashkin-Teller and 8-vertex models [49-51].

The variation in the exponents occurs over a range of connectivities that is significantly larger than that found in collagen networks. Therefore, one can use a unique set of exponents, $f \simeq 0.8$ and $\phi \simeq 2.1$, to describe the mechanics of collagen networks prepared at different concentrations [23]. The uniqueness of the exponents also allows us to relate the two characteristic strains of a subisostatic network, onset of stiffening strain, and critical strain via the critical exponents as $\gamma_{0} \sim \gamma_{c}^{\phi-f}$.

In sum, the mechanics of disordered fibrous networks can be understood within the framework of an athermal strain-driven critical phenomenon. The mechanical criticality is a generic phenomenon exhibited by all subisostatic networks. We apply our model to collagen networks, which are ubiquitous in biology, and find strong evidence for the idea that mechanical critical behavior underlies the strain-stiffening response of collagenous networks.
[1] P. Fratzl, Collagen: Structure and Mechanics (Springer Science \& Business Media, Berlin, 2008).

[2] J. Clerk Maxwell F.R.S., Philos. Mag. Ser. 4 27, 294 (1864).

[3] A. J. Liu and S. R. Nagel, Nature 396, 21 (1998).

[4] T. S. Majmudar, M. Sperl, S. Luding, and R. P. Behringer, Phys. Rev. Lett. 98, 058001 (2007).

[5] M. Van Hecke, J. Phys.: Condens. Matter 22, 033101 (2010).

[6] A. J. Liu, S. R. Nagel, W. van Saarloos, and M. Wyart, in Dynamical Heterogeneities in Glasses, Colloids, and Granular Media, edited by L. Berthier, G. Biroli, J.-P. Bouchaud, L. Cipeletti, and W. van Saarloos (Oxford University Press, Oxford, 2010), pp. 298-336.

[7] M. F. Thorpe, J. Non-Cryst. Solids 57, 355 (1983).

[8] S. Feng and P. N. Sen, Phys. Rev. Lett. 52, 216 (1984).

[9] D. J. Jacobs and M. F. Thorpe, Phys. Rev. Lett. 75, 4051 (1995).

[10] M. Latva-Kokko, J. Mäkinen, and J. Timonen, Phys. Rev. E 63, 046113 (2001).

[11] P. Olsson and S. Teitel, Phys. Rev. Lett. 99, 178001 (2007).

[12] D. A. Head, Phys. Rev. Lett. 102, 138001 (2009).

[13] M. Wyart, H. Liang, A. Kabla, and L. Mahadevan, Phys. Rev. Lett. 101, 215501 (2008).

[14] W. G. Ellenbroek, Z. Zeravcic, W. van Saarloos, and M. van Hecke, Europhys. Lett. 87, 34004 (2009).

[15] C. P. Broedersz, X. Mao, T. C. Lubensky, and F. C. MacKintosh, Nature Phys. 7, 983 (2011).

[16] M. Sheinman, C. P. Broedersz, and F. C. MacKintosh, Phys. Rev. E 85, 021801 (2012).

[17] A. J. Licup, A. Sharma, and F. C. MacKintosh, Phys. Rev. E 93, 012407 (2016).
[18] D. A. Head, A. J. Levine, and F. C. MacKintosh, Phys. Rev. Lett. 91, 108102 (2003).

[19] J. Wilhelm and E. Frey, Phys. Rev. Lett. 91, 108103 (2003).

[20] M. Sheinman, C. P. Broedersz, and F. C. MacKintosh, Phys. Rev. Lett. 109, 238101 (2012).

[21] M. Dennison, M. Sheinman, C. Storm, and F. C. MacKintosh, Phys. Rev. Lett. 111, 095503 (2013).

[22] S. Alexander, Phys. Rep. 296, 65 (1998).

[23] A. Sharma, A. Licup, K. Jansen, R. Rens, M. Sheinman, G. Koenderink, and F. MacKintosh, Nature Phys. 12, 584 (2016).

[24] C. P. Broedersz and F. C. MacKintosh, Soft Matter 7, 3186 (2011).

[25] C. P. Broedersz, M. Sheinman, and F. C. MacKintosh, Phys. Rev. Lett. 108, 078102 (2012).

[26] X. Mao, O. Stenull, and T. C. Lubensky, Phys. Rev. E 87, 042602 (2013).

[27] D. A. Head, A. J. Levine, and F. C. MacKintosh, Phys. Rev. E 68, 061907 (2003).

[28] E. M. Huisman, T. van Dillen, P. R. Onck, and E. Van der Giessen, Phys. Rev. Lett. 99, 208103 (2007).

[29] A. Shahsavari and R. C. Picu, Phys. Rev. E 86, 011923 (2012).

[30] E. Conti and F. C. MacKintosh, Phys. Rev. Lett. 102, 088102 (2009).

[31] A. Sharma, M. Sheinman, K. M. Heidemann, and F. C. MacKintosh, Phys. Rev. E 88, 052705 (2013).

[32] K. M. Heidemann, A. Sharma, F. Rehfeldt, C. F. Schmidt, and M. Wardetzky, Soft Matter 11, 343 (2015).

[33] A. J. Licup, S. Münster, A. Sharma, M. Sheinman, L. M. Jawerth, B. Fabry, D. A. Weitz, and F. C. MacKintosh, Proc. Natl. Acad. Sci. USA 112, 9573 (2015). 
[34] D. A. Head, F. C. MacKintosh, and A. J. Levine, Phys. Rev. E 68, 025101 (2003).

[35] A. S. van Oosten, M. Vahabi, A. J. Licup, A. Sharma, P. A. Galie, F. C. MacKintosh, and P. A. Janmey, Scientific Reports 6, 19270 (2016).

[36] M. Vahabi, A. Sharma, A. J. Licup, A. S. van Oosten, P. A. Galie, P. A. Janmey, and F. C. MacKintosh, Soft matter 12, 5050 (2016).

[37] M. Achilli and D. Mantovani, Polymers 2, 664 (2010).

[38] M. S. Hall, R. Long, X. Feng, Y. Huang, C.-Y. Hui, and M. Wu, Exp. Cell Res. 319, 2396 (2013).

[39] R. Picu, Soft Matter 7, 6768 (2011).

[40] S. Motte and L. J. Kaufman, Biopolymers 99, 35 (2013).

[41] I. K. Piechocka, A. S. van Oosten, R. G. Breuls, and G. H. Koenderink, Biomacromolecules 12, 2797 (2011).

[42] J. P. Straley, J. Phys. C 9, 783 (1976).

[43] H. Hatami-Marbini and R. C. Picu, Phys. Rev. E 77, 062103 (2008).

[44] A. Arrott and J. E. Noakes, Phys. Rev. Lett. 19, 786 (1967).

[45] N. Goldenfeld, Lectures on Phase Transitions and the Renormalization Group (Addison-Wesley, Advanced Book Program, Reading, 1992).

[46] C. P. Broedersz and F. C. MacKintosh, Rev. Mod. Phys. 86, 995 (2014).
[47] D. Stauffer and A. Aharony, Introduction to Percolation Theory (CRC Press, Boca Raton, FL, 1994).

[48] R. Rens, M. Vahabi, A. J. Licup, F. C. MacKintosh, and A. Sharma, J. Phys. Chem. B 120, 5831 (2016).

[49] J. Ashkin and E. Teller, Phys. Rev. 64, 178 (1943).

[50] R. J. Baxter, Phys. Rev. Lett. 26, 832 (1971).

[51] L. P. Kadanoff and A. C. Brown, Ann. Phys. 121, 318 (1979).

[52] N. P. Butch and M. B. Maple, Phys. Rev. Lett. 103, 076404 (2009).

[53] D. Fuchs, M. Wissinger, J. Schmalian, C.-L. Huang, R. Fromknecht, R. Schneider, and H. v. Löhneysen, Phys. Rev. B 89, 174405 (2014).

[54] T. Xu, D. Vavylonis, F.-C. Tsai, G. H. Koenderink, W. Nie, E. Yusuf, I.-J. Lee, J.-Q. Wu, and X. Huang, Sci. Rep. 5, 9081 (2015).

[55] R. C. Arevalo, P. Kumar, J. S. Urbach, and D. L. Blair, PLOS One 10, e0118021 (2015).

[56] M. L. Gardel, J. H. Shin, F. C. MacKintosh, L. Mahadevan, P. A. Matsudaira, and D. A. Weitz, Science 304, 1301 (2004).

[57] C. Storm, J. Pastore, F. C. MacKintosh, T. C. Lubensky, and P. A. Janmey, Nature 435, 191 (2005).

[58] K. E. Kasza, G. H. Koenderink, Y. C. Lin, C. P. Broedersz, W. Messner, F. Nakamura, T. P. Stossel, F. C. MacKintosh, and D. A. Weitz, Phys. Rev. E 79, 041928 (2009). 